\title{
Temperature dependent photoluminescence of lateral polarity junctions of metal organic chemical vapor deposition grown GaN
}

Cite as: J. Appl. Phys. 110, 093503 (2011); https://doi.org/10.1063/1.3656987

Submitted: 19 June 2011 . Accepted: 22 September 2011 . Published Online: 02 November 2011

Ronny Kirste, Ramón Collazo, Gordon Callsen, Markus R. Wagner, Thomas Kure, Juan Sebastian Reparaz, Seji Mita, Jinqiao Xie, Anthony Rice, James Tweedie, Zlatko Sitar, and Axel Hoffmann

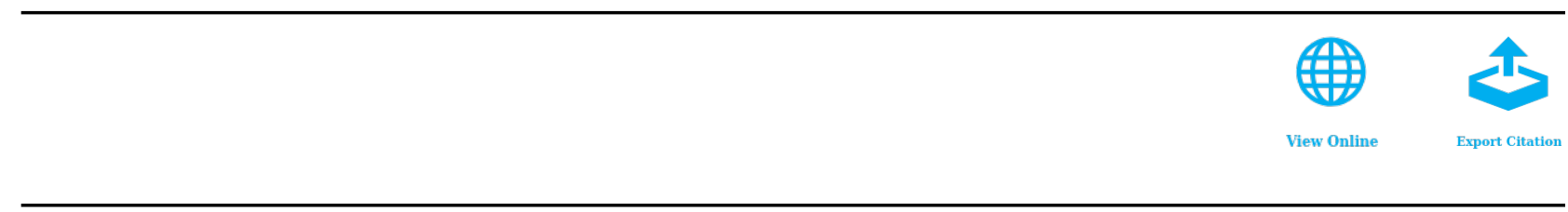

\section{ARTICLES YOU MAY BE INTERESTED IN}

Polarity control and growth of lateral polarity structures in AIN

Applied Physics Letters 102, 181913 (2013); https://doi.org/10.1063/1.4804575

The mechanism for polarity inversion of GaN via a thin AIN layer: Direct experimental evidence Applied Physics Letters 91, 203115 (2007); https://doi.org/10.1063/1.2815748

Luminescence properties of defects in GaN

Journal of Applied Physics 97, 061301 (2005); https://doi.org/10.1063/1.1868059

Lock-in Amplifiers Find out more today

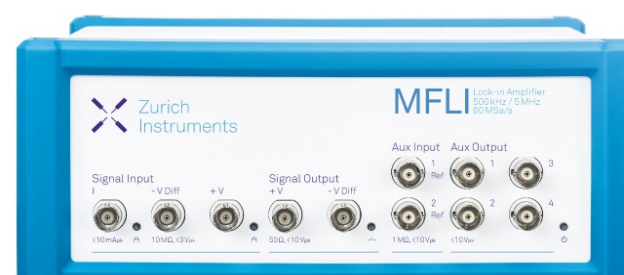

Zurich - Instruments 


\title{
Temperature dependent photoluminescence of lateral polarity junctions of metal organic chemical vapor deposition grown GaN
}

\author{
Ronny Kirste, ${ }^{1, a)}$ Ramón Collazo, ${ }^{2}$ Gordon Callsen, ${ }^{1}$ Markus R. Wagner, ${ }^{1}$ Thomas Kure, ${ }^{1}$ \\ Juan Sebastian Reparaz, ${ }^{1}$ Seji Mita, ${ }^{3}$ Jinqiao Xie, ${ }^{3}$ Anthony Rice, ${ }^{2}$ James Tweedie, ${ }^{2}$ \\ Zlatko Sitar, ${ }^{2}$ and Axel Hoffmann ${ }^{1}$ \\ ${ }^{1}$ Institute of Solid State Physics, Technische Universität Berlin, Hardenbergstrasse 36, D-10623 Berlin, Germany \\ ${ }^{2}$ Department of Materials Science and Engineering, North Carolina State University, Raleigh, \\ North Carolina 27695, USA \\ ${ }^{3}$ HexaTech, Inc., 991 Aviation Pkwy., Suite 800, Morrisville, North Carolina 27560, USA
}

(Received 19 June 2011; accepted 22 September 2011; published online 2 November 2011)

\begin{abstract}
We report on fundamental structural and optical properties of lateral polarity junctions in GaN. GaN with Ga- to N-polar junctions was grown on sapphire using an AlN buffer layer. Results from scanning electron microscopy and Raman spectroscopy measurements indicate a superior quality of the Ga-polar GaN. An extremely strong luminescence signal is observed at the inversion domain boundary (IDB). Temperature dependent micro photoluminescence measurements are used to reveal the recombination processes underlying this strong emission. At $5 \mathrm{~K}$ the emission mainly arises from a stripe along the inversion domain boundary with a thickness of 4-5 $\mu \mathrm{m}$. An increase of the temperature initially leads to a narrowing to below $2 \mu \mathrm{m}$ emission area width followed by a broadening at temperatures above $70 \mathrm{~K}$. The relatively broad emission area at low temperatures is explained by a diagonal IDB. It is shown that all further changes in the emission area width are related to thermalization effects of carriers and defects attracted to the IDB. The results are successfully used to confirm a theoretical model for GaN based lateral polarity junctions. Due to the strong and pronounced emission of IDBs even at elevated temperatures, it is demonstrated that lateral polarity junctions exhibit a strong potential for future high efficiency devices. (C) 2011 American Institute of Physics. [doi:10.1063/1.3656987]
\end{abstract}

\section{INTRODUCTION}

$\mathrm{GaN}$ and its related ternary alloys $\mathrm{InGaN}$ and $\mathrm{AlGaN}$ are the leading materials for photonic devices in the visible and ultra violet wavelength regime. Extremely important for high efficient photonic devices is a deep understanding of recombination centers in these materials. One can distinguish between radiative and non-radiative recombination centers. Nonradiative recombination centers such as threading dislocations have to be avoided as they reduce the external quantum efficiency. ${ }^{1}$ The controlled incorporation of radiative recombination centers is a good possibility for high efficient light-emitting diodes (LEDs). Most of these radiative recombination centers are introduced by the incorporation of defects. These could be structural defects or dopants as for example In atoms in $\mathrm{GaN}^{2}{ }^{2}$ Unfortunately, the incorporation of such desired recombination centers often goes hand in hand with the introduction of non-radiative recombination centers.

Nearly one decade ago Schuck et al. presented laterally resolved photoluminescence measurements on the inversion domain boundary (IDB) in GaN. ${ }^{3}$ They revealed that at the $\mathrm{N}$-polar to Ga-polar junction in $\mathrm{GaN}$, an enhancement of the luminescence signal of one order of magnitude is observed. Further calculations by Fiorentini explained this strong emission of the IDB by a maximum of the local potential in the core of the IDB accompanied by two local minima that can effectively collect electrons. ${ }^{4}$ It was suggested that optical

${ }^{a)}$ Electronic mail: rkirste@physik.tu-berlin.de. devices based on such polar junctions could lead to a new generation of optical devices. ${ }^{5}$ In the following years only few publications dealing with the optical, electrical, and structural properties of IDBs were published, ${ }^{6,7}$ which might be related to difficulties in the controlled growth of lateral polarity junctions (LPJs). For that reason many properties of polar junctions are still unknown. But for the implementation of IDBs into optical devices, a better understanding of the recombination processes is indispensible.

In this contribution we present fundamental investigations of the structural and optical properties of lateral polarity junctions in GaN. Scanning electron microscopy and Raman spectroscopy are used to explain the results obtained by temperature dependent micro photoluminescence. This data is not available in literature, so far and is needed to understand the processes underlying the strong luminescence of the IDB. The model calculated by Fiorentini is confirmed and extended. ${ }^{4}$ Since the signal of the inversion domain is still very strong at room temperature, it is demonstrated that polar junctions are extremely interesting for next generation optical devices.

\section{EXPERIMENT}

GaN was grown in a low pressure metal organic chemical vapor deposition (MOCVD) reactor at temperatures around $1030^{\circ} \mathrm{C}$. Area selective growth of Ga- and N-polar $\mathrm{GaN}$ was achieved via the laterally controlled growth of a low temperature (LT) AlN buffer layer on sapphire substrate 
and subsequent nitridization. Details of the growth process can be found elsewhere. ${ }^{8}$ Consequently, in the border between the $\mathrm{N}$ - and Ga-polar GaN, lateral polarity junctions were grown; thereby the inversion domain boundary (IDB) was accessible for optical investigations from the surface. By growing samples under different V/III ratios, which corresponds to different supersaturations, a selective incorporation of carbon was achieved leading to different carrier concentrations and mobilities. ${ }^{9}$ In this contribution we focus on two samples: Sample A grown under a V/III ratio of around 100 and sample B under a V/III ration of approximately 2000. Scanning electron microscopy (SEM) and cathodoluminescence (CL) investigations were undertaken with a FEI Quanta 200 ESEM, which was equipped with a MONOCL3 System by Gatan. It was the main aim of those investigations to gain information about the sample's surface morphology and the deep defect related luminescence. Room temperature micro Raman spectroscopy measurements were performed using a DILOR XY 800 triple grating Raman spectrometer with the $488 \mathrm{~nm}$ line of an argon ion laser as excitation source. In order to obtain information about the lateral distribution of the crystal properties the setup was coupled to a XY-table, which enabled map scans with a resolution down to $500 \mathrm{~nm}$. The near band edge photoluminescence (PL) was examined with a micro photoluminescence ( $\mu$-PL) setup allowing map scans with a resolution down to $300 \mathrm{~nm}$. Thereby, the $325 \mathrm{~nm}$ line of a HeCd laser was used as excitation source, the PL signal was dispersed using a $0.45 \mathrm{~m}$ single monochromator and detected by a nitrogen cooled CCD with $2048 \times 512$ pixel. For temperature dependent measurements, an Oxford constant flow cryostat allowed a variation of the temperature in the range from 5 to $300 \mathrm{~K}$.

\section{RESULTS}

Figure 1 displays SEM images of the IDB for sample A (left) and B (right). For both samples the surface of the $\mathrm{N}$-polar region is very rough, as it is dominated by hexagonal pyramids well aligned to the crystal axis. The size of these pyramids varies from $1 \mu \mathrm{m}$ up to $8 \mu \mathrm{m}$ in diameter. No pyramids are observed in the Ga-polar region where the surface is flat and nearly free of observable structural defects. Such pyramidal structures in N-polar GaN are not unusual and have been observed before. ${ }^{5,10}$ Collazo et al. pointed out that the roughness of the $\mathrm{N}$-polar regions can be reduced by
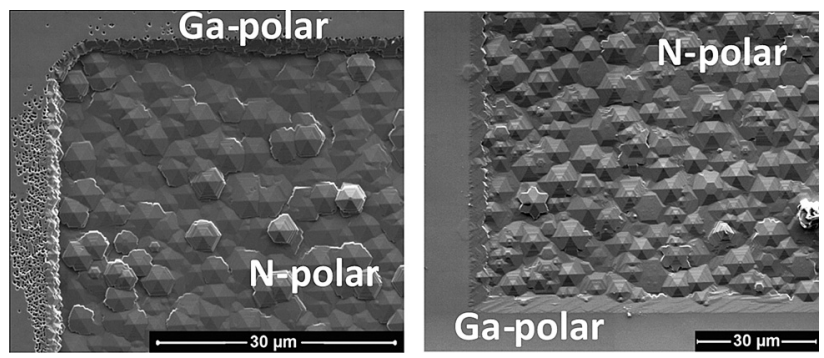

FIG. 1. SEM images of the inversion domain boundary (Ga-polar to $\mathrm{N}$-polar junction) for sample A (left) and B (right). The N-polar regions are dominated by pyramidal structures while the Ga-polar regions reveal a mainly flat surface. longer nitridization times of the substrate up to a level where it is comparable to that of the Ga-polar region. ${ }^{8}$ In our case a distinct roughness of the $\mathrm{N}$-polar region was chosen on purpose in order to simplify the orientation and alignment of the crystal.

In the past, different models for the IDB with monoatomic inversion domains were suggested. ${ }^{11,12}$ However, TEM measurements by Mita et al. revealed, that due to the imprecise etching of the LT-AIN layer the LPJ consists of around $50 \mathrm{~nm}$ with mixed polarity. ${ }^{13}$ Taking into account our results obtained by SEM, a much broader IDB for our samples seems to be obvious. In Fig. 1 a step-like shape between the N-polar and Ga-polar area is visible. This step has a width of around $1.5 \mu \mathrm{m}$ for sample A and $2.5 \mu \mathrm{m}$ for sample B, whereby the origin of the different thickness is attributed to the different V/III ratios while growth. These findings are confirmed by atomic force microscopy (AFM) measurements. Thereby, a step with a width comparable to that revealed by the SEM images and a height of $600-800 \mathrm{~nm}$ between the two polar areas was observed (not shown). This vertical step is caused by a thicker Ga-polar region compared to the N-polar region. Taking a closer look at Fig. 1, overgrowth of N-polar GaN is observed, since some of the pyramids in the N-polar region are half covered by smooth Ga-polar GaN. Thus, the inversion domain boundary is not expected to be perpendicular to the surface.

A schematic illustration of the polarity junction is shown in Fig. 2. The angle under which the inversion domain is grown increases the effective (observable) area of the IDB, viewable from a top view, to a few micrometers. A rough estimation of the angle between the inversion domain and the substrate delivers a value of 50-60 degree. Following the results obtained by SEM/AFM, we estimate the effective IDB-width to $1.5 \mu \mathrm{m}$ for sample A and $2.5 \mu \mathrm{m}$ for sample B.

Different incorporation of defects depending on the polarity is well known for many materials including GaN, InN, and $\mathrm{ZnO} .{ }^{14-16}$ It has been shown that in many cases the surface with the polarity of the cation has a much lower unintentional doping rate compared to that of the anion side. ${ }^{17}$ Cathodoluminescence spectra of the N- and Ga-polar areas of sample A were recorded in order to confirm these findings and are shown in Fig. 3. The spectra were normalized to the near band edge emission (NBE). Thereby, it

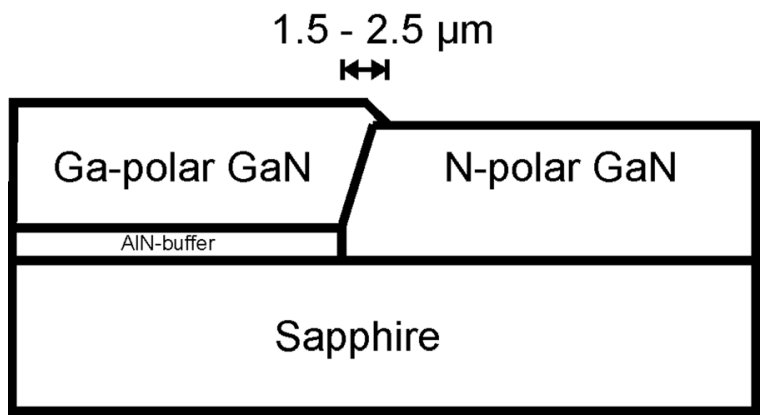

FIG. 2. Illustration of the expected sample-structure in the area of the inversion domain. The IDB lies under a distinct angle and is not perpendicular to the surface. This leads to an increased effective IDB area of $1.5-2.5 \mu \mathrm{m}$, whereby the effective area shall be defined as the area observable from a top view. The thickness of the AlN-buffer is roughly $50 \mathrm{~nm}$, the thickness of the $\mathrm{GaN}$ film is supposed to be $2 \mu \mathrm{m}$. 


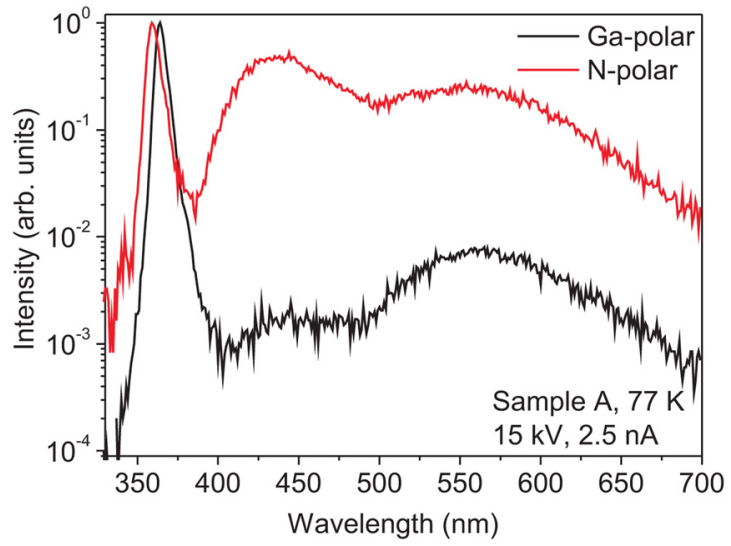

FIG. 3. (Color online) Cathodoluminescence spectra of the Ga- and N-polar region of sample A. Spectra were normalized to the near band edge luminescence.

should be mentioned that the intensity of the NBE of the Gapolar region was enhanced by a factor of 15 compared to that of the N-polar region. This result is consistent with earlier results and explained by different non-radiative recombination rates. ${ }^{3}$ Further discussion of the NBE will follow below when results of photoluminescence measurements are taken into account. Additionally to the NBE, two strong defect luminescence bands are observed. While the broad peak centered between $400 \mathrm{~nm}$ and $500 \mathrm{~nm}$, the so-called blue luminescence, is normally attributed to $\mathrm{Mg}, \mathrm{Si}$, or $\mathrm{Zn}$ incorporation, ${ }^{18}$ the origin of the yellow luminescence between 500 and $600 \mathrm{~nm}$ is attributed to $\mathrm{Ga}$ vacancies or oxygen impurities. Compared to the NBE, the defect luminescence of the Ga-polar area is much weaker than that of the $\mathrm{N}$-polar area indicating decreased defect incorporation. In the $\mathrm{N}$-polar area the blue luminescence is nearly as strong as the near band edge luminescence while it is more than two orders of magnitude weaker in the Ga-polar area. Although the intensity of the yellow luminescence also decreases, its reduction is smaller. This is in good agreement with results published in $\mathrm{ZnO}$, where a secondary ion mass spectrometry (SIMS) analysis of $\mathrm{Zn}$ - and O-polar samples revealed a reduced impurity concentration of up to two orders of magnitude in the Zn-face samples. ${ }^{17}$ It is of importance to confirm these findings with our samples since the investigation of different surface polarities is normally performed on two different samples. Although grown in the same growth-run, a spatial displacement could result in different gas flows, a temperature gradient and so on, which would lead to slightly different growth conditions. For samples with a LPJ, this can be excluded due to the fact that the spatial displacement is only a few micrometers.

In order to assess the strain, crystal quality and electronic properties of the GaN epilayers in the IDB area, laterally resolved Raman spectra were recorded with a step width of $1 \mu \mathrm{m}$ over a square of $20 \times 20 \mu \mathrm{m}$. Spectra were taken in $\mathrm{z}(\mathrm{xx}) z$ geometry, consequently the $\mathrm{E}_{2}$ (high) and $\mathrm{A}_{1}(\mathrm{LO})$ Raman modes are allowed. ${ }^{19}$ The line shapes of these modes were analyzed and the position and full width at half maximum (FWHM) was plotted laterally resolved in Figs. 4(a)- 4(d) for the $E_{2}$ (high) for both samples and in Figs. 4(e) $+4(f)$ for the $\mathrm{A}_{1}(\mathrm{LO})$ mode of sample $\mathrm{B}$. The $\mathrm{E}_{2}$ (high) mode is non-polar and thus only slightly influenced by carriers in the crystal. Therefore, it can be used to evaluate the strain in the layers. Taking into account the relaxed position of the $\mathrm{E}_{2}$ (high) mode at $567 \mathrm{~cm}^{-1}$, it can be seen that the $\mathrm{N}$-polar regions for both samples are relaxed while the Ga-polar regions exhibit a small compressive strain. ${ }^{19}$ These different strain states can be easily understood if the SEM and CL results presented above are taken into account. For the N-polar region pyramidal structures were observed indicating a strong 3D growth, which leads to relaxation. On the other hand, the Ga-polar region exhibited a smooth surface with few structural defects, which leads to a small strain originating from the lattice mismatch between substrate and layer. The FWHM of the $\mathrm{E}_{2}$ (high) mode is an indicator for the crystal quality. Comparing the Ga-polar to the $\mathrm{N}$ polar region an increase from around $3 \mathrm{~cm}^{-1}$ to $8 \mathrm{~cm}^{-1}$ in sample $A$ and to $5 \mathrm{~cm}^{-1}$ in sample $B$ is observed. While $3 \mathrm{~cm}^{-1}$ is a good value for epitaxial grown $\mathrm{GaN}, 5-8 \mathrm{~cm}^{-1}$ is an indicator for a decreased crystal quality. The driving force for the decreased crystal quality is supposed to be the increased defect incorporation in the N-polar region as observed with CL. Thus, the results obtained by SEM and CL are confirmed and the superior crystal quality of the Ga-polar region is demonstrated by Raman measurements.

The inversion domain is well established in Figs. 4(a)4(f) as a vertical line between the two areas of different polarity. But this visibility is only due to the strain and quality gradient between the $\mathrm{N}$ - and Ga-polar area. The IDB itself cannot be observed, except in Fig. 4(d) where a stripe like feature is seen. In contrast to the $\mathrm{E}_{2}$ (high) mode, the position and FWHM of the $\mathrm{A}_{1}(\mathrm{LO})$ mode are not exclusively influenced by the strain but also by charge carriers and their mobility. At higher carrier concentrations, the phonons might couple to the carrier plasma that leads to a broadening of this mode and a shift to higher energies. ${ }^{20}$ Both, the position as well as the FWHM of the $\mathrm{A}_{1}(\mathrm{LO})$ mode are displayed in Fig. $4(\mathrm{e})+4(\mathrm{f})$. Comparing the Ga-polar and N-polar region, a shift of the position from $735 \mathrm{~cm}^{-1}$ to $738 \mathrm{~cm}^{-1}$ and a broadening from $6 \mathrm{~cm}^{-1}$ to $9 \mathrm{~cm}^{-1}$ are observed indicating an increased carrier concentration in the N-polar region. ${ }^{20}$ This behavior was observed before and is explained by a polarity dependent incorporation of oxygen. ${ }^{21,22}$ Again, no differentiation between the inversion domain and the N-polar or Gapolar region is observed. Taking into account the effective IDB-width of $1.5-2.5 \mu \mathrm{m}$, this is very striking, since the polarity junction is supposed to be accomplished by a potential minimum that effectively collects carriers. ${ }^{4}$ A possible explanation could be the measurement at room temperature, whereby thermalization could activate carriers and allow them to drift away from the IDB into the Ga-/N-polar region.

For a detailed analysis of the recombination processes at the inversion domain, $\mu$-PL spectra were recorded in an area of $20 \times 20 \mu \mathrm{m}$ with a step width of $330 \mathrm{~nm}$. Thereby, the inversion domain was a vertical line in the center, the Ga-polar area on the left side and the N-polar region on the right side. Typical PL spectra of the IDB and both polar areas are displayed in Fig. 5. The spectra were recorded at $5 \mathrm{~K}$. Drastic differences between the three regions are observed regarding the position of the main peak as well as their intensity. It should be mentioned that also some 

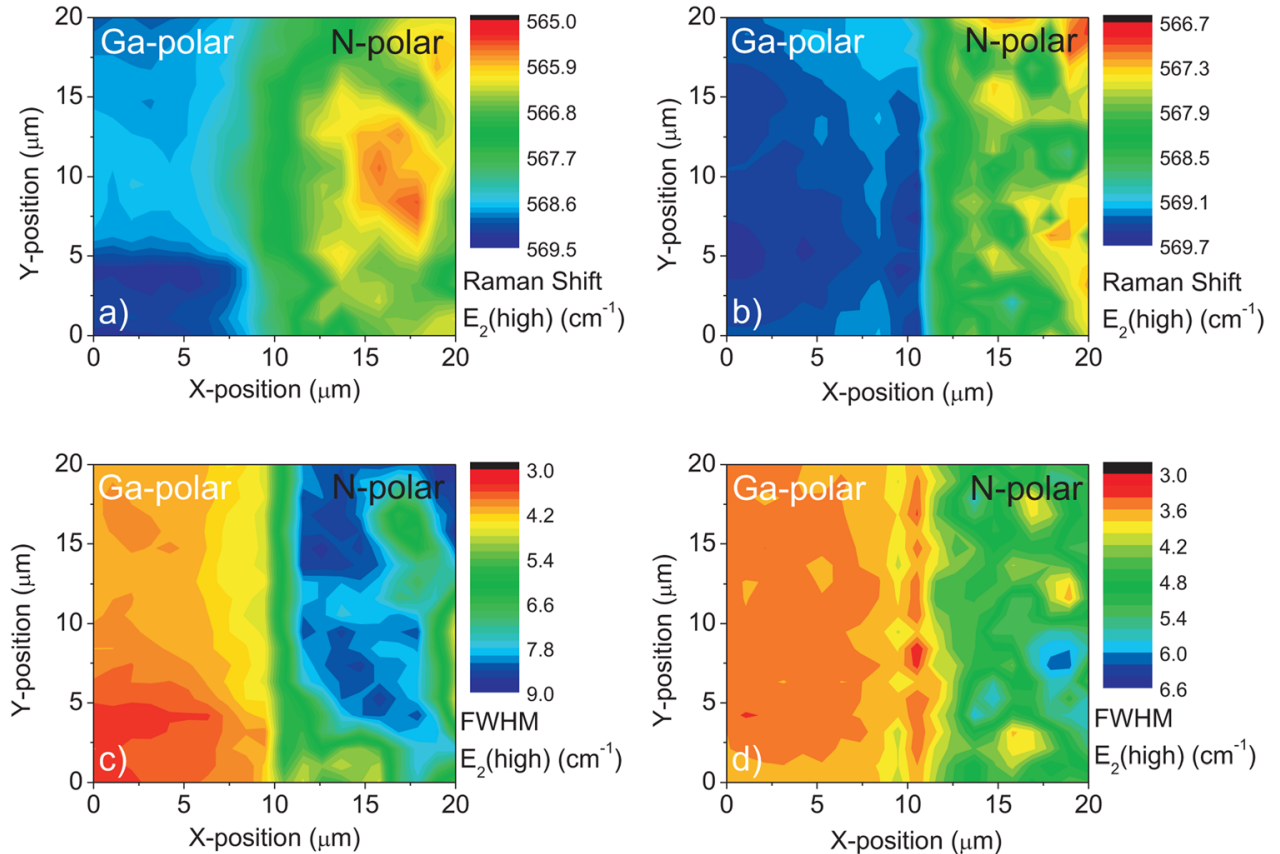

FIG. 4. (Color online) 2D maps in the area of the IDB of the position of the $\mathrm{E}_{2}$ (high) mode (a),(b) and its FWHM (c), (d) for sample A (left) and sample B (right), as well as position (e) and FWHM (f) of the $A_{1}(L O)$ mode for sample B.
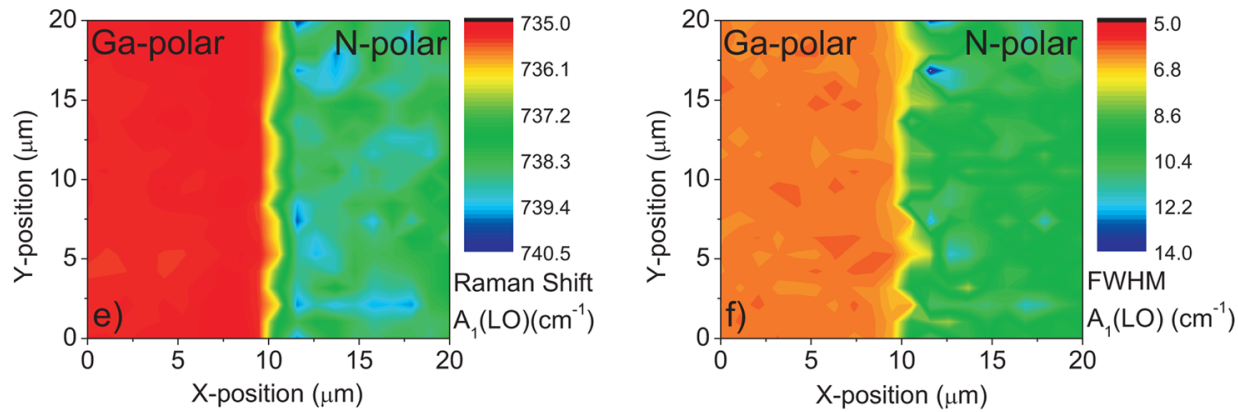

variations between the spectra of each distinct region are observed. These fluctuations are attributed to varying defect incorporation and are small compared to those between the Ga-/N-polar and ID region. The spectra taken in the Gapolar area exhibit features, which are generally observed for n-type GaN. The peaks at $3.493 \mathrm{eV}, 3.485 \mathrm{eV}$, and $3.478 \mathrm{eV}$ are attributed to the B-exciton, A-exciton and donor bound exciton, ${ }^{18}$ which is confirmed by the temperature dependent behavior of these lines (not shown). All peaks are shifted

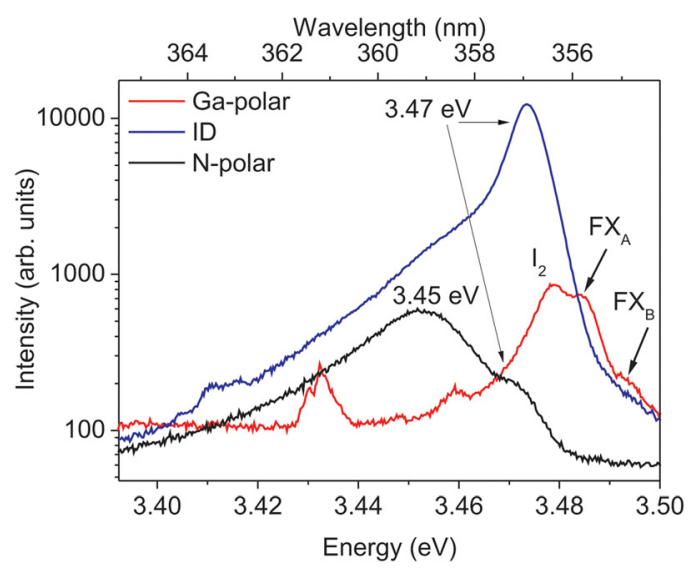

FIG. 5. (Color online) Typical low temperature (5 K) PL spectra of the $\mathrm{N}$-polar GaN, Ga-polar GaN and the inversion domain area of sample A.
$8 \mathrm{meV}$ to higher energies compared to their values in relaxed GaN. This shift is due to the compressive strain in the Ga-polar region as it was observed by Raman spectroscopy (Fig. 4). ${ }^{23}$ In the N-polar area two broad peaks at around $3.45 \mathrm{eV}$ and $3.47 \mathrm{eV}$ are observed. Reshchikov et al. ascribed a line at $3.42-3.43 \mathrm{eV}$ to structural defects near the surface. ${ }^{18}$ Although the emission of the N-polar region is observed at higher energies, it is also attributed to structural defects. SEM results presented in Fig. 1 support this interpretation where a high density of pyramids was found in the N-polar area. A strong shift for the emission of such pyramids was previously observed. Liu et al. performed laterally resolved cathodoluminescence in $\mathrm{GaN}$ pyramids and revealed a strong redshift of $40 \mathrm{meV}$ from the top to the base of these pyramids due to a strong tensile strain. ${ }^{23}$ Taking these results into account the high FWHM of the emission of the N-polar region is explained by a strain gradient that cannot be observed with Raman spectroscopy or $\mu$-PL due to the limited spatial resolution of the setup. Thus, the increased FWHM of the $E_{2}$ (high) mode in the N-polar region is not only interpreted as a decreased crystal quality but also as result of a strain gradient in the pyramidal structures (Figs. 4(c) and 4(d)). The maximum of the emission of the IDB is found between those of the N-/Ga-polar regions at $3.47 \mathrm{eV}$. This is about $20 \mathrm{meV}$ above the values for IDB emission as published by Schuck et al. ${ }^{3}$ The argumentation for this shift 
is comparable to that for the shift of the emission of the Npolar area. As revealed by SEM measurements and illustrated in Fig. 2, the inversion domain consists mainly of a boundary grown under a distinct angle to the surface. The border lies between unstrained N-polar GaN and compressively strained Ga-polar GaN. Hence, a strong strain field affects the IDB signal, whereby the compressive strain originating in the Ga-polar region dominates and leads to a shift to higher energies. The intensities of the signals of the Ga- and N-polar regions are of comparable height. In contrast, the signal of the ID is more than one order of magnitude stronger. This behavior was observed before by Schuck et al. with a comparable intensity ratio. ${ }^{3}$ Fiorentini discussed the origin of this efficient light emission and suggested a model with a potential maximum in the center of the ID accomplished by two minima in the $\mathrm{N}-/ \mathrm{Ga}$-polar regions. ${ }^{4} \mathrm{~A}$ detailed analysis of this model will be discussed below. A closer look at Fig. 5 reveals, that the IDB related signal not only consists of the signal at $3.47 \mathrm{eV}$ but has also a low and a high energy shoulder. A line shape analysis reveals that energetic position of the shoulders is very close to the values of the N- and Ga polar region, respectively. Thus, we identify the origin of these shoulders as the emission of the underlying N-/Ga-polar GaN (Fig. 2).

Figure 6 shows laterally resolved integrated intensity images of the near band edge luminescence of both samples
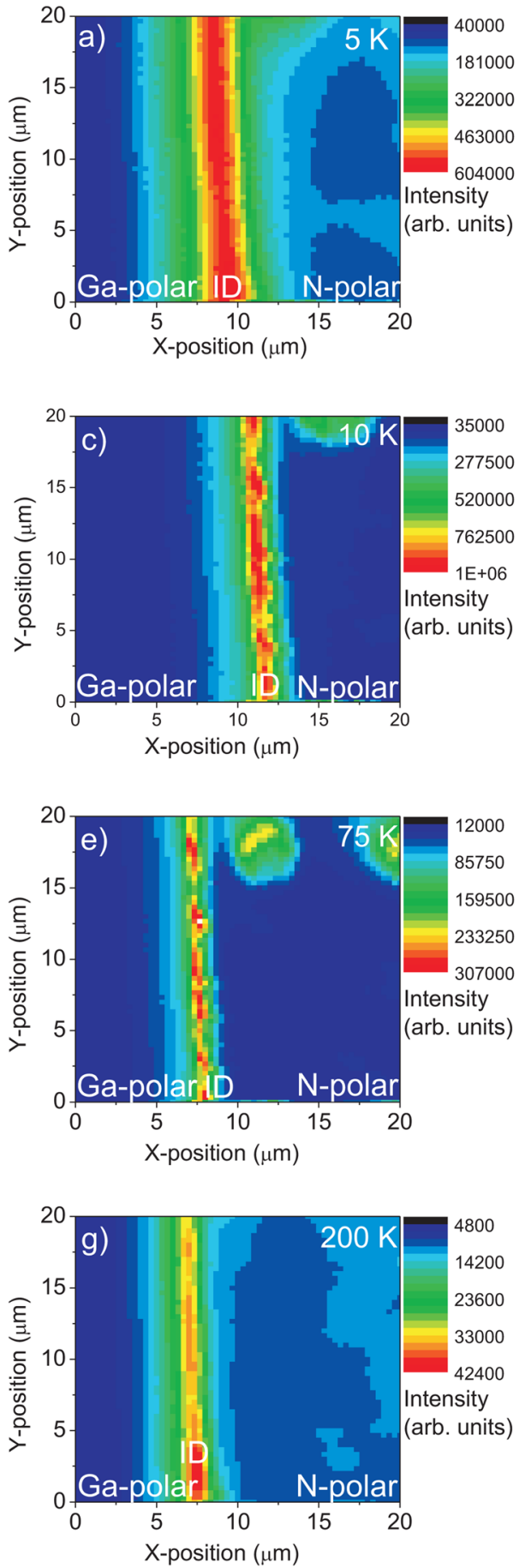
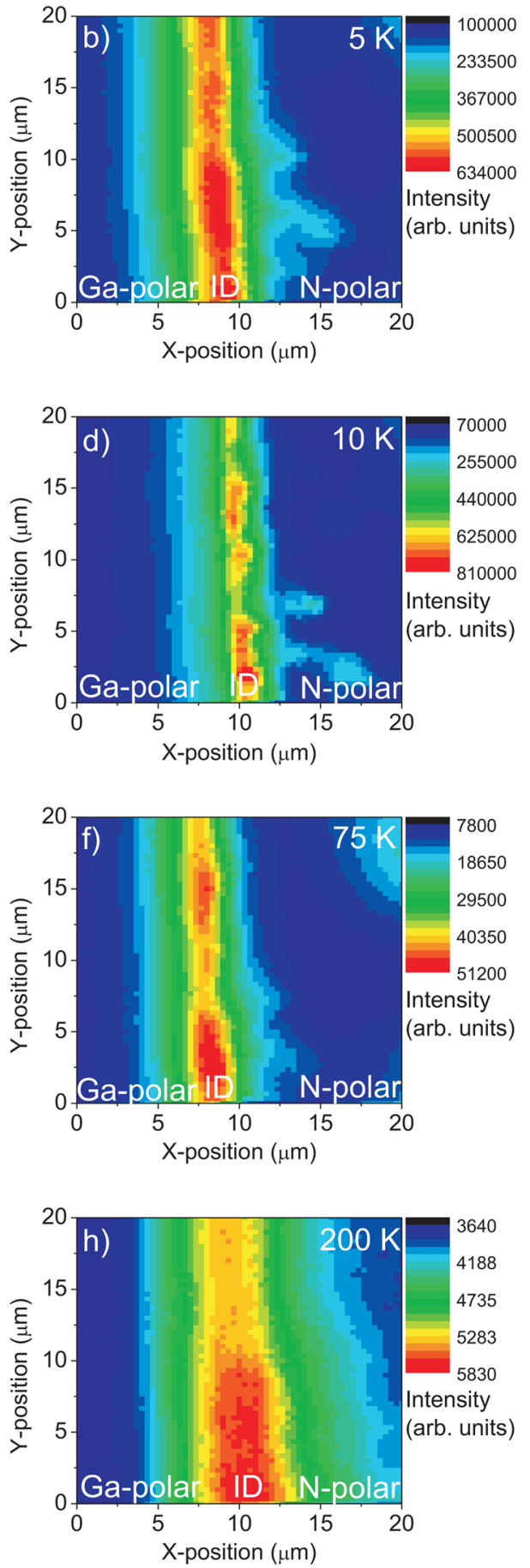

FIG. 6. (Color online) Laterally resolved integrated intensity $\mu$-PL images of the NBE luminescence in the area of the inversion domain boundary for sample A (left) and B (right). The displayed 2D maps were recorded at $5 \mathrm{~K}, 10 \mathrm{~K}, 75 \mathrm{~K}$, and $200 \mathrm{~K}$ (top to bottom). 
for different temperatures. The alignment of the samples for these measurements was chosen so that the IDB was oriented as a vertical line in the center of the investigated area. At all temperatures the main emission comes from the IDB, revealed by the highest integral intensity. This is in accordance with results published by Schuck et $a .^{3}$ In their samples no PL-signal was detected in the N-polar regions while a signal one order of magnitude lower than that of the IDB was observed in the Ga-polar regions. As mentioned in the discussion of Fig. 5, the observed intensity ratio between the Ga-polar signal and IDB signal at $5 \mathrm{~K}$ is in good agreement to that of Schuck et al. However, it was also shown that a signal of similar intensity to that of the Ga-polar region can be seen in the N-polar region. Furthermore, for some temperatures the integral intensity of the $\mathrm{N}$-polar region is even stronger than that of the Ga-polar area, especially in sample A. As revealed by SEM, CL, and Raman spectroscopy the N-polar area consists of pyramidal structures with a high defect concentration. A reasonable explanation for the emission of the N-polar region is that these defects act as strong optical recombination centers.

The character of the stripe-like emission of the IDB is strongly temperature dependent. At $5 \mathrm{~K}$ the thickness of this emission area is similar in both samples and lies around $4.5 \mu \mathrm{m}$, which is higher than the width of the IDB as observed by SEM. If the temperature is raised from $5 \mathrm{~K}$ to $10 \mathrm{~K}$ a diminution of the emission area of the IDB related signal to $2 \mu \mathrm{m}$ in sample $\mathrm{A}$ and $3 \mu \mathrm{m}$ in sample $\mathrm{B}$ is seen. Furthermore, an increase of the overall intensity in that region is observed as for example in sample $A$ from $6.0 \times 10^{5}$ to $1.0 \times 10^{6}$. A further rise of the temperature leads to a small broadening of the emission area in the case of sample A and a much higher increase in the case of sample B. Additionally, the integral intensity of all areas decreases drastically for higher temperatures.

\section{DISCUSSION}

By using $\Delta$ self-consistent field (SCF) principle, Fiorentini calculated the local potential in the inversion domain as depicted in Fig. 7.4 The strong emission of the IDB is explained by a very high potential centered in the IDB core, which is accompanied by two local minima on each of its sides. In this model the electrons are captured separately in the two local minima while the holes are trapped in a local maximum in the vicinity of the IDB. Taking into account the

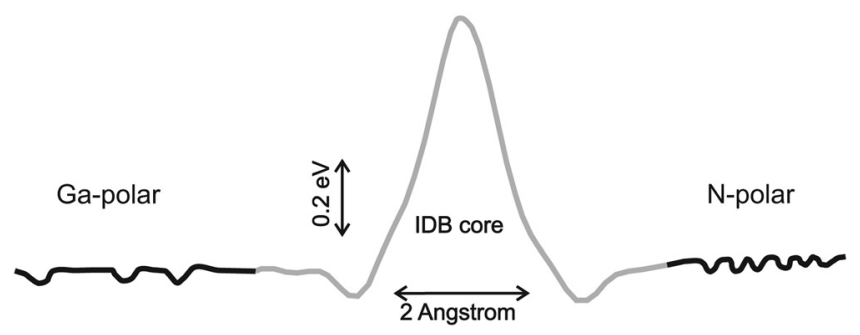

FIG. 7. Model for the local potential as calculated by Fiorentini (light gray). ${ }^{4}$ The potential maximum is accompanied by two local minima in each polar area leading to strong excitonic emission. Additionally, local minima at both sides of the IDB due to defect states are suggested (black). theoretical models for the ID predicting the change from Ga-polar to N-polar within one atomic layer, a strong overlap of the electron and hole wave functions is expected. ${ }^{11,12}$ This can be also seen in the calculations of Fiorentini as the size of the IDB core is only a few angstrom. An interesting result is the height of the IDB local potential. A rough estimation from the publication of Fiorentini generates a value of $75 \mathrm{meV}$ for the local minima and around $600 \mathrm{meV}$ for the maximum compared to the height of the surrounding potential. These values are remarkably high if one takes into account typical binding energies of excitons (26 meV). Thereby, it should be mentioned that the calculations of Fiorentini could neither reproduce the observed energetic shift of $30 \mathrm{meV}$ of the undisturbed cell nor identify if the emission of the IDB is of excitonic character.

The model of Fiorentini is used to explain the experimental results obtained by temperature dependent photoluminescence (Fig. 6). Thereto, this model is complemented by local minima close to the IDB as depicted in Fig. 7. Origins of the local minima are defects that are collected by the IDB. This explanation is very likely taking into account results known from other structural defects as for example threading dislocation that effectively collect point defects. ${ }^{24}$ It can be seen in Fig. 7 that fewer defects are assumed in the Ga-polar region compared to the $\mathrm{N}$-polar region. This is based on results obtained by SEM, CL, and Raman spectroscopy that clearly revealed decreased structural quality and increased defect incorporation of the $\mathrm{N}$-polar region caused by pyramidal structures.

At low temperatures $(\leq 5 \mathrm{~K})$ a strong $\mathrm{PL}$ signal is observed along the IDB region (Fig. 6). Thereby, the main emission area is a stripe with a width of 4-5 $\mu \mathrm{m}$ depending on the sample. A temperature rise initially leads to a decrease of the emission area width followed by a broadening for temperatures above $75 \mathrm{~K}$. In order to analyze this behavior in detail, single lines of the laterally resolved integral intensity maps were extracted. Two typical linescans are displayed in Fig. 8. Line shapes of all single lines were fitted and the FWHM as well as the intensity distribution were noted. It can be seen in Fig. 8 that the line shape of the intensity is asymmetrical. In order to find an accurate fit, different model were applied, whereby it was found that a Fano line shape is the best fitting model for most lines. The FWHM of the Fano line shape depending on the temperature is displayed in Fig. 9. It can be clearly seen that the temperature dependent behavior of the emission area of the IDB, as described above, is well reproduced by the FWHM. This behavior is explained by thermalization of carriers trapped by defects near the IDB (Fig. 7). At $5 \mathrm{~K}$ and below the main emission originates from the IDB, which is broadened compared to the value observed by SEM (Fig. 1). Carriers that are excited close to the IDB will be collected by the local potential extreme of the polar junction and recombine there, leading to the observed high emission intensity. However, some of the carriers will be also trapped by defects close to the IDB. Due to the low temperature they do not have sufficient thermal energy to escape from these defects, thus, they will recombine, not in the IDB, but at the defects. If the temperature is increased to $10-20 \mathrm{~K}$ the FWHM of the emission area is reduced down to 


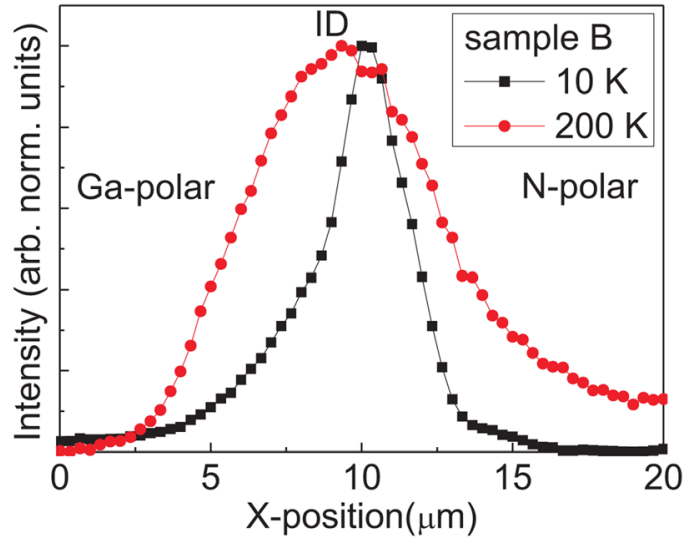

FIG. 8. (Color online) Linescans of the integral intensity maps in Figs. 6(d) and $6(\mathrm{~h})$ - the PL intensity depending on the $\mathrm{x}$-position is shown for two different temperatures. The IDB is in the center. The fit of these line shapes delivers the FWHM of the IDB as displayed in Fig. 9.

1.5-2 $\mu \mathrm{m}$, which is comparable to the step width observed by SEM and AFM. At these temperatures, carriers that are excited close to the polar junction and are trapped by the defects have enough thermal energy to escape from there. Thus, they will migrate to the IDB where they finally recombine. This leads to a smaller emission area and increased intensity, compared to that at $5 \mathrm{~K}$ (Figs. 6 and 9). The thermalization effect dominates the temperature dependent behavior up to $70 \mathrm{~K}$. For these temperatures the FWHM of the emission area stays nearly constant. A further increase of the temperature leads again to a broadening. This broadening is small for sample A but very pronounced for sample B. Origin is a thermalization from the IDB. Fiorentini calculated the depth of the local minimum for the IDB to around $75 \mathrm{meV},{ }^{4}$ which exceeds the thermal energy at room temperature $(26 \mathrm{meV})$ by a factor of three. Thus, not all carriers are activated; some are still trapped in the IDB, which can be proven by the fact that the emission of the IDB is still well pronounced with higher intensities than that of the Ga-/N-polar regions. It was mentioned that the width of the emission area decreases to $1.5 \mu \mathrm{m}$ in sample $\mathrm{A}$, which is in fact the

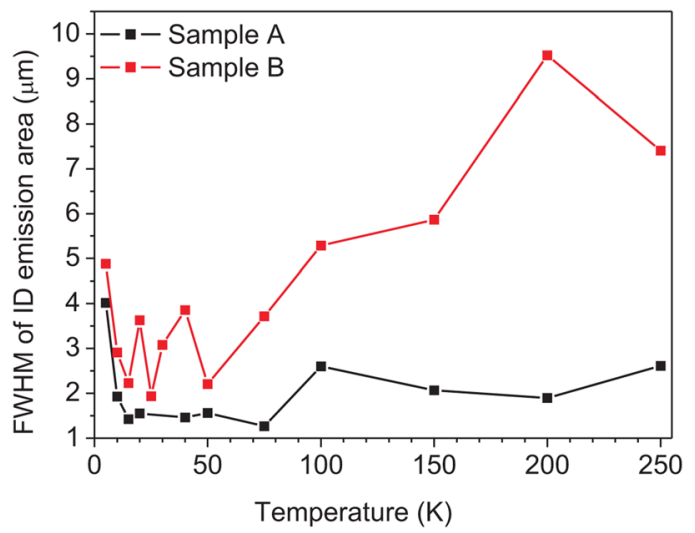

FIG. 9. (Color online) Full width at half maximum of the emission area of samples $\mathrm{A}$ and $\mathrm{B}$ depending on the temperature. The values are the mean values from the fit of the plots of the PL-intensities depending on the $\mathrm{X}$-position as depicted in Fig. 8. The solid lines are a guide to the eyes. value observed by SEM. In contrast, for sample $B$ the FWHM decreases down to $1.9 \mu \mathrm{m}$, which is even below the width obtained by SEM. At all temperatures the emission area of sample B is always much broader than that of sample A. To attribute this broadening only to the different width of the IDB is insufficient, especially at higher temperatures. More likely, this effect can be explained by the different charge carrier mobility of the samples due to the applied growth conditions. It was shown that a higher Ga supersaturation rate during growth leads to a decreased carbon incorporation and, thus, no compensation of unintentionally incorporated donors. ${ }^{9}$ For that reason, the Ga-polar region of sample B has a higher carrier concentration and mobility than that of sample A. Since the carrier mean free path is proportional to the mobility, thermally activated carriers in sample B are able to propagate further away from the IDB in sample B than in sample A.

In Fig. 8 two typical linescans of the integral intensity depending on the distance to the IDB at $10 \mathrm{~K}$ and $200 \mathrm{~K}$ are displayed. It was mentioned that the peaks had to be fitted with a Fano line shape due to their asymmetry. This asymmetry is very remarkable as it is a strong indicator for the model proposed by Fiorentini. ${ }^{4}$ He proposed a local maximum in the center of the IDB surrounded by two local minima on each side of the polar junction. This local maximum divides the emission area into two separated regions; a Ga-polar and a N-polar related. The density of defects that can trap carriers is much lower in the Ga-polar than in the $\mathrm{N}$-polar region. At low temperatures $(\mathrm{T}<10 \mathrm{~K})$ carriers once trapped by a defect cannot thermalize from it, as described above. This leads to the tail of the intensity in the Ga-polar side, as carriers excited there can drift much more apart from the IDB than in the N-polar region. At elevated temperatures $(\mathrm{T}>70 \mathrm{~K})$ carriers can escape from the defect states through thermal energy. Thus, the defect density is no longer the limiting factor. For these temperatures the limiting factor is only the mobility, which is higher in the N-polar region. Consequently, the tail of the asymmetrical Fano line extends into the N-polar side of the IDB.

In order to confirm the defect model suggested in Fig. 7 excitation power dependent photoluminescence measurements were performed. For that purpose, the excitation source for micro PL was changed from a HeCd laser to the fourth harmonic of a pulsed Nd:YAG laser $(266 \mathrm{~nm}, 76 \mathrm{MHz}$ repetition rate, $60 \mathrm{ps}$ pulse length) as it allows much higher power densities. Excitation power dependent measurements were performed with $2 \mathrm{~mW}$ and $0.2 \mathrm{~mW}$. In order to enable a simple comparison of the map scans, the excitation power density was increased by one order of magnitude while the integration time was reduced by one order of magnitude. Figure 10 displays the integral intensity in the area of the IDB for two different excitation powers at $5 \mathrm{~K}$. Analyzing the FWHM of the emission area, a width of the IDB of $5 \mu \mathrm{m}$ is revealed for the lower excitation power. This value is above that of map scans obtained with the $\mathrm{HeCd}$ laser as excitation source (Fig. 9), which is related to the pulsed excitation and the following high excitation density. A further rise of the excitation power density leads to an increase of the IDB width to $5.8 \mu \mathrm{m}$. Additionally, the intensity of the 

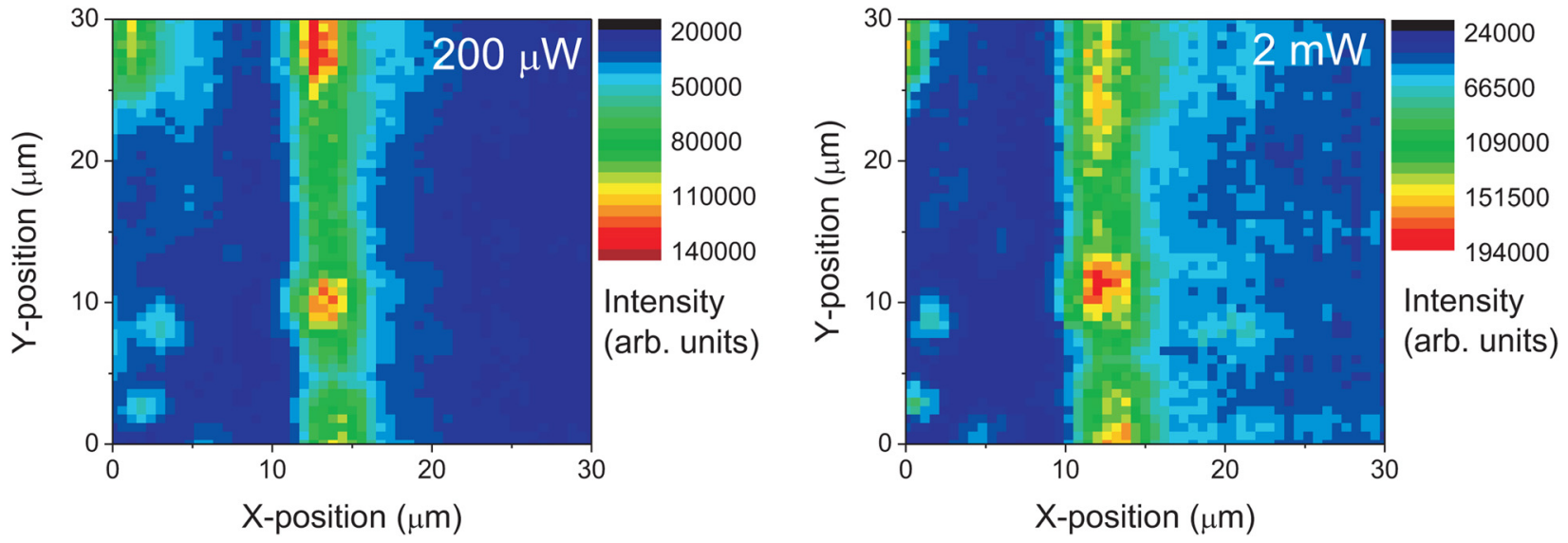

FIG. 10. (Color online) Laterally resolved integrated intensity $\mu$-PL images of the near band edge luminescence in the area of the inversion domain boundary for sample $\mathrm{A}$ at $5 \mathrm{~K}$ for different excitation densities.

emission of the Ga- and N-polar region is enhanced, whereby the increase is much more pronounced in the $\mathrm{N}$-polar region. The broadening as well as the increasing intensity of luminescence in the polar regions is attributed to a higher carrier concentration due to the elevated excitation power density. This higher carrier concentration leads to a saturation of the defects close to the IDB. Carriers trapped in such local minima that have not enough thermal energy to escape from these defects can escape much easier if the potential is filled. Thus, they can migrate to other defects and recombine there. The higher intensity in the N-polar compared to the Ga-polar region is a strong indicator for a higher defect density, which leads to the model as described in Fig. 7.

\section{SUMMARY}

In summary the recombination processes at the Ga-polar to $\mathrm{N}$-polar inversion domain boundaries in GaN were investigated by temperature dependent photoluminescence measurements. Preliminary structural and optical investigations revealed a strong defect incorporation in the N-polar compared to the Ga-polar GaN. By SEM imaging a pyramidal structure was observed in the $\mathrm{N}$-polar region that was in contrast to the smooth surface of the Ga-polar GaN. Low temperature micro photoluminescence measurements revealed a strong emission of the IDB. At $5 \mathrm{~K}$ the width of the emission area width of the IDB related signal is $4-5 \mu \mathrm{m}$. A slight increase of temperature from $5 \mathrm{~K}$ to $10 \mathrm{~K}$ leads to a decreased FWHM of the emission area combined with an increased intensity. A further rise leads to a drop of the intensity and a broadened emission area. The intensive emission of the polar junction itself is explained by a maximum of the local potential in the core of the IDB, which is accompanied by two local minima in the N-/Ga-polar region as calculated by Fiorentini. A strong evidence for such a strong local potential that can disturb the free movement of carriers is the integral intensity of the luminescence, which exhibited an asymmetric line shape. The drop of the emission area width for slightly increased temperatures is explained by a thermalization of carriers that are effectively collected by defects near the IDB and may migrate toward the polar junction. It is shown that a further increase of the temperature leads to the activation of carriers from the IDB, leading to an apparent broadening at elevated temperatures. Finally, the strong and pronounced emission of the IDB at high temperatures reveals the opportunities of LPJs for high efficient light emitting devices.

\section{ACKNOWLEDGMENTS}

Parts of this work were supported by DFG within the SFB 787.

${ }^{1}$ T. Sugahara, H. Sato, M. S. Hao, Y. Naoi, S. Kurai, S. Tottori, K. Yamashita, K. Nishino, L. T. Romano, and S. Sakai, Jpn. J. Appl. Phys. Part 2 Lett. 37, L398 (1998).

${ }^{2}$ S. F. Chichibu, K. Wada, J. Mullhauser, O. Brandt, K. H. Ploog, T. Mizutani, A. Setoguchi, R. Nakai, M. Sugiyama, H. Nakanishi, K. Korii, T. Deguchi, T. Sota, and S. Nakamura, Appl. Phys. Lett. 76, 1671 (2000).

${ }^{3}$ P. J. Schuck, M. D. Mason, R. D. Grober, O. Ambacher, A. P. Lima, C. Miskys, R. Dimitrov, and M. Stutzmann, Appl. Phys. Lett. 79, 952 (2001). ${ }^{4}$ V. Fiorentini, Appl. Phys. Lett. 82, 1182 (2003).

${ }^{5}$ M. Stutzmann, O. Ambacher, M. Eickhoff, U. Karrer, A. Lima Pimenta, R. Neuberger, J. Schalwig, R. Dimitrov, P. Schuck, and R. Grober, Phys. Status Solidi B 228, 505 (2001).

${ }^{6}$ M. Niebelschutz, G. Ecke, V. Cimalla, K. Tonisch, and O. Ambacher, J. Appl. Phys. 100, 074909 (2006).

${ }^{7}$ F. Liu, R. Collazo, S. Mita, Z. Sitar, S. J. Pennycook, and G. Duscher, Adv. Mater. 20, 2162 (2008).

${ }^{8}$ R. Collazo, S. Mita, A. Aleksov, R. Schlesser, and Z. Sitar, J. Cryst. Growth 287, 586 (2006).

${ }^{9}$ S. Mita, R. Collazo, A. Rice, R. F. Dalmau, and Z. Sitar, J. Appl. Phys. 104, 013521 (2008).

${ }^{10}$ H. M. Ng, N. G. Weimann, and A. Chowdhury, J. Appl. Phys. 94, 650 (2003).

${ }^{11}$ J. E. Northrup, J. Neugebauer, and L. T. Romano, Phys. Rev. Lett. 77, 103 (1996).

${ }^{12}$ L. T. Romano, J. E. Northrup, and M. A. O'Keefe, Appl. Phys. Lett. 69, 2394 (1996).

${ }^{13}$ F. Liu, R. Collazo, S. Mita, Z. Sitar, G. Duscher, and S. J. Pennycook, Appl. Phys. Lett. 91, 203115 (2007).

${ }^{14}$ F. Tuomisto, K. Saarinen, B. Lucznik, I. Grzegory, H. Teisseyre, T. Suski, S. Porowski, P. R. Hageman, and J. Likonen, Appl. Phys. Lett. 86, 031915 (2005).

${ }^{15}$ R. Kirste, M. R. Wagner, J. H. Schulze, A. Strittmatter, R. Collazo, Z. Sitar, M. Alevli, N. Dietz, and A. Hoffmann, Physica Status Solidi A 207, 2351 (2010).

${ }^{16}$ M. R. Wagner, T. P. Bartel, R. Kirste, A. Hoffmann, J. Sann, S. Lautenschlager, B. K. Meyer, and C. Kisielowski, Phys. Rev. B 79, 035307 (2009).

${ }^{17}$ S. Lautenschlaeger, J. Sann, N. Volbers, B. K. Meyer, A. Hoffmann, U. Haboeck, and M. R. Wagner, Phys. Rev. B 77, 144108 (2008).

${ }^{18}$ M. A. Reshchikov and H. Morkoc, J. Appl. Phys. 97, 061301 (2005). 
${ }^{19}$ U. Haboeck, H. Siegle, A. Hoffmann, and C. Thomsen, Phys. Status Solidi C, 0(6), 1710 (2003).

${ }^{20}$ P. Perlin, J. Camassel, W. Knap, T. Taliercio, J. C. Chervin, T. Suski, I. Grzegory, and S. Porowski, Appl. Phys. Lett. 67, 2524 (1995).

${ }^{21}$ M. Park, J. J. Cuomo, B. J. Rodriguez, W. C. Yang, R. J. Nemanich, and O. Ambacher, J. Appl. Phys. 93, 9542 (2003).
${ }^{22}$ T. K. Zywietz, J. Neugebauer, and M. Scheffler, Appl. Phys. Lett. 74, 1695 (1999).

${ }^{23}$ Q. K. K. Liu, A. Hoffmann, H. Siegle, A. Kaschner, C. Thomsen, J. Christen, and F. Bertram, Appl. Phys. Lett. 74, 3122 (1999).

${ }^{24}$ J. Elsner, R. Jones, M. I. Heggie, P. K. Sitch, M. Haugk, T. Frauenheim, S. Öberg, and P. R. Briddon, Phys. Rev. B 58, 12571 (1998). 